\title{
Scanning Tunneling Spectroscopy of Ag Films: The Effect of Periodic versus Quasiperiodic Modulation
}

\author{
Daejin Eom, ${ }^{1}$ C.-S. Jiang, ${ }^{1}$ H.-B. Yu, ${ }^{1}$ J. Shi, ${ }^{2}$ Q. Niu, ${ }^{1}$ Ph. Ebert, ${ }^{3}$ and C.-K. Shih ${ }^{1}$ \\ ${ }^{1}$ Department of Physics, The University of Texas, Austin, Texas 78712, USA \\ ${ }^{2}$ Institute of Physics and ICQS, Chinese Academy of Sciences, Beijing 100080, China \\ ${ }^{3}$ Institut für Festkörperforschung, Forschungszentrum Jülich GmbH, 52425 Jülich, Germany
}

(Received 21 February 2006; published 16 November 2006)

\begin{abstract}
By using scanning tunneling spectroscopy to probe a silver thin film that contains both periodic and quasiperiodic modulation, and by using Fourier analysis, we unravel the influences of individual Fourier components of the scattering potential (periodic versus quasiperiodic) on the electronic structure of a onedimensional quasiperiodically modulated thin Ag film. Along the periodically modulated direction, a Bragg reflection-induced energy gap is observed in $k$ space. On the other hand, the exotic $E$ vs $k$ spectrum with many minigaps was observed along the quasiperiodic direction.
\end{abstract}

PACS numbers: 73.20.- r, 61.44.Br, 68.37.Ef

When electrons move in crystalline solids, the coherent scattering with the periodic potential enables them to sneak by all ions uninhibited, forming Bloch electrons with their own $E$ vs $k$ dispersion relationship. In quasicrystals, however, such a "Bloch description" of the electronic structure encounters conceptual difficulties, due to the absence of periodicity. Instead, theoretical investigations predicted exotic characteristics such as the Cantor-set energy spectrum [1]. However, experiments did not confirm such exotic properties [2]. Angle-resolved photoemission measurement of the $E$ vs $k$ dispersion relation in a quasicrystal rather revealed free-electron-like states [3]. Nevertheless, critical questions remain as to how electronic states in a quasiperiodic solid differ from those in a periodic solid.

The central question lies in the fact that the atomic arrangement of quasicrystal is not periodic yet possesses a long range order. The lack of translational periodicity renders the inapplicability of Bloch's theorem. One can no longer define a Brillouin zone for a quasicrystal in its strict meaning, because the diffraction pattern has densely distributed reciprocal vectors in $\boldsymbol{k}$ space [1,4]. The concept of electronic bands also fails, since the energy spectrum of a quasicrystal is predicted to form a Cantor set and gaps are dense in the spectrum [4-7]. Nevertheless, "effective" descriptions are often found to be useful depending on the experimental conditions such as the apparatus' resolution limit or the Zener breakdown between energy states $[8,9]$. For example, bandlike features have been recently observed in the $E$ vs $k$ dispersion relation probed by angleresolved photoemission on decagonal Al-Ni-Co quasicrystals [3]. The observed effective electronic bands resemble free-electron-like ones, suggesting that the electronic states are not localized in nature but rather exhibit appreciable amounts of delocalized character. On the other hand, anomalies in certain physical properties of quasicrystals have also been reported [10-12]. Still, some critical questions remained unanswered. In particular, what are the roles of densely distributed reciprocal vectors in $\boldsymbol{k}$ space? Here we tackle this problem directly by studying the electronic structure of a thin Ag film whose surface contains both quasiperiodically and periodically modulated potentials. This feature allows us to compare directly the manifestation of quasiperiodicity versus periodicity within the same experiment. In addition, as the electronic structures are probed locally using scanning tunneling spectroscopy (STS), the spatial averaging effect masking fine electronic structures is completely avoided. The $k$-resolved electronic structures are obtained through a Fourier analysis, following the so-called Fourier-transformed STS [13,14].

Heavily $p$-doped GaAs wafers were cleaved in ultrahigh vacuum $\left(p<1 \times 10^{-10}\right.$ mbar $)$ to expose (110) surfaces. $\mathrm{Ag}$ was deposited from a thermal evaporator on substrates cooled to $100 \mathrm{~K}$. Subsequently, the samples were annealed to RT to form atomically flat Ag films [15], which were then investigated in situ in a scanning tunneling microscope (STM) operated at about $6 \mathrm{~K}$ with a thermal drift smaller than $1 \mathrm{~nm} /$ day.

Figure 1(a) shows a typical STM image of such a Ag film. Oval-shaped features (called "ovals" hereafter) are arranged in rows parallel to the GaAs [110] direction (vertical or $\hat{y}$ ). The spacing $h$ between two ovals along $\hat{y}$ is $2.8 \AA$, the same as the interatomic spacing along the bulk silver [1 $1 \overline{1} 0]$ direction. Furthermore, along $\hat{y}$ there is an additional weak modulation (typical corrugation of only $0.08 \AA$ ) with a periodicity of $11 \AA$ (corresponding to $3.7 \times$ h) [Fig. 1(b)]. Along the GaAs [001] direction (horizontal or $\hat{x}$ ), the rows of ovals exhibit distinct widths and separations as marked in Fig. 1(a) $(q=5.1 \AA, p=7.4 \AA, S=$ $12.5 \AA$, and $L=17.6 \AA$ ). The much larger corrugation of $0.6 \AA$ is coupled to the $S$ and $L$ separations. Figure 1 (c) shows a large field of view where only rows with the $S$ and $L$ separations are clearly noticeable. As analyzed previously, the $S$ and $L$ feature sizes are arranged in real space following the Fibonacci sequence, albeit the existence of 

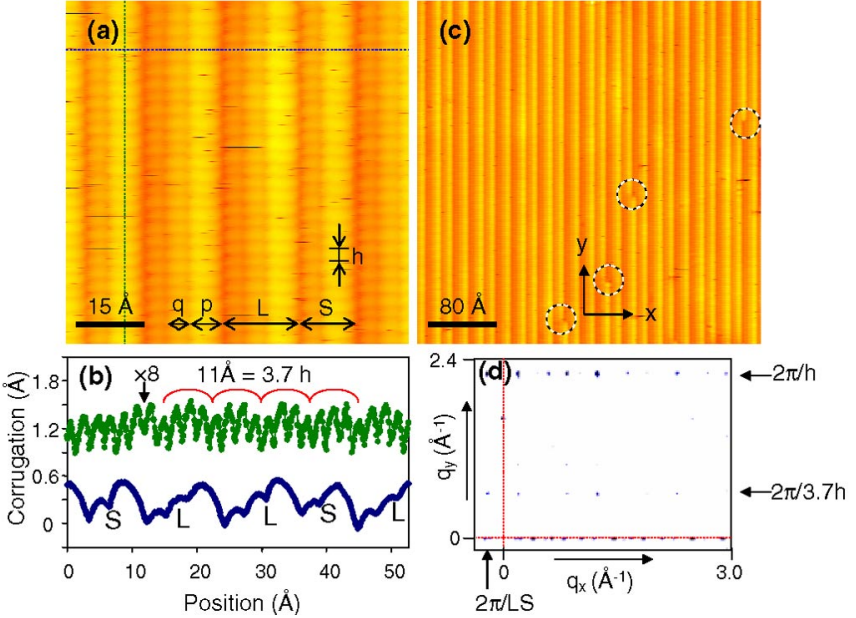

FIG. 1 (color online). (a) STM images of Ag film (film thickness $18 \AA$ ) grown on a GaAs (110) substrate are acquired at $6.8 \mathrm{~K}$ with different lateral dimensions. (b) Topographic line profiles along the lateral (blue) and vertical (green) directions, respectively. (c) A larger STM image of the same film showing the phason defects (marked by the dashed circles). (d) A quarter section of the 2D Fourier transformation of the topographic image shown in (c).

phason defects (see dashed circles) often disrupts the ideal sequence [16]. Figure 1(d) shows the Fourier transformation of the topographic image in Fig. 1(c) where the effects of periodic and quasiperiodic modulations are shown along $q_{y}$ and $q_{x}$, respectively. The particular characteristic of a periodic modulation along $\hat{y}$ in conjunction with a quasiperiodic modulation along $\hat{x}$ has provided us with an excellent opportunity to directly compare the effect of a quasiperiodic potential vs a periodic potential on the electronic structure.
Figures 2(b)-2(e) show four differential conductance maps acquired at different biases $V_{b}$ applied to the sample, along with the topographic image [Fig. 2(a)]. These conductance maps are proportional to the local density of states (LDOS) $\rho(E, x, y)$ at the energy $E=e V_{b}$ [17]. Before analyzing the conductance maps, we recall that the states below $E_{F}$ are quantum well states whose structures depend on the film thickness [18]. However, the electronic states above $E_{F}$, and notably the most prominent one directly above $E_{F}$, are independent of the film thickness and attributed to Shockley-type surface states whose scattering with the periodic and the quasiperiodic potentials is of interest here.

The conductance image at $E_{F}+1.83 \mathrm{eV}$ [Fig. 2(b)] shows similar structures as the topography. The dominant features are the stripe patterns along the vertical direction. In contrast, the conductance maps at $E_{F}+0.95 \mathrm{eV}$ and $E_{F}+0.55 \mathrm{eV}$ [Figs. 2(c) and 2(d)] both exhibit a mesh network as a consequence of a fine structure along $\hat{y}$, which has, however, a different wavelength in both images. The conductance image at $E_{F}+0.04 \mathrm{eV}$ [Fig. 2(e)] exhibits again a stripe pattern along the vertical direction, which is, however, enhanced for long wavelengths compared to that of the topography [Fig. 2(a)]. The conductance line profiles measured along the dotted lines in $\hat{x}$ as a function of energy [Fig. 2(f)] illustrate additional information. Also shown in Fig. 2(g) are two representative $d I / d V$ vs $V$ spectra acquired at two neighboring locations, one on the ridge and the other one on the valley of topography [labeled in Fig. 2(a)], illustrating spatial dependence of the tunneling spectrum. While all line profiles display quasiperiodic modulations, the actual modulation patterns vary as a function of energy: For example, the conductance profile at $E_{F}+1.83 \mathrm{eV}$ is almost identical to the topographic profile, whereas the profile at $E_{F}+0.95 \mathrm{eV}$ has a phase

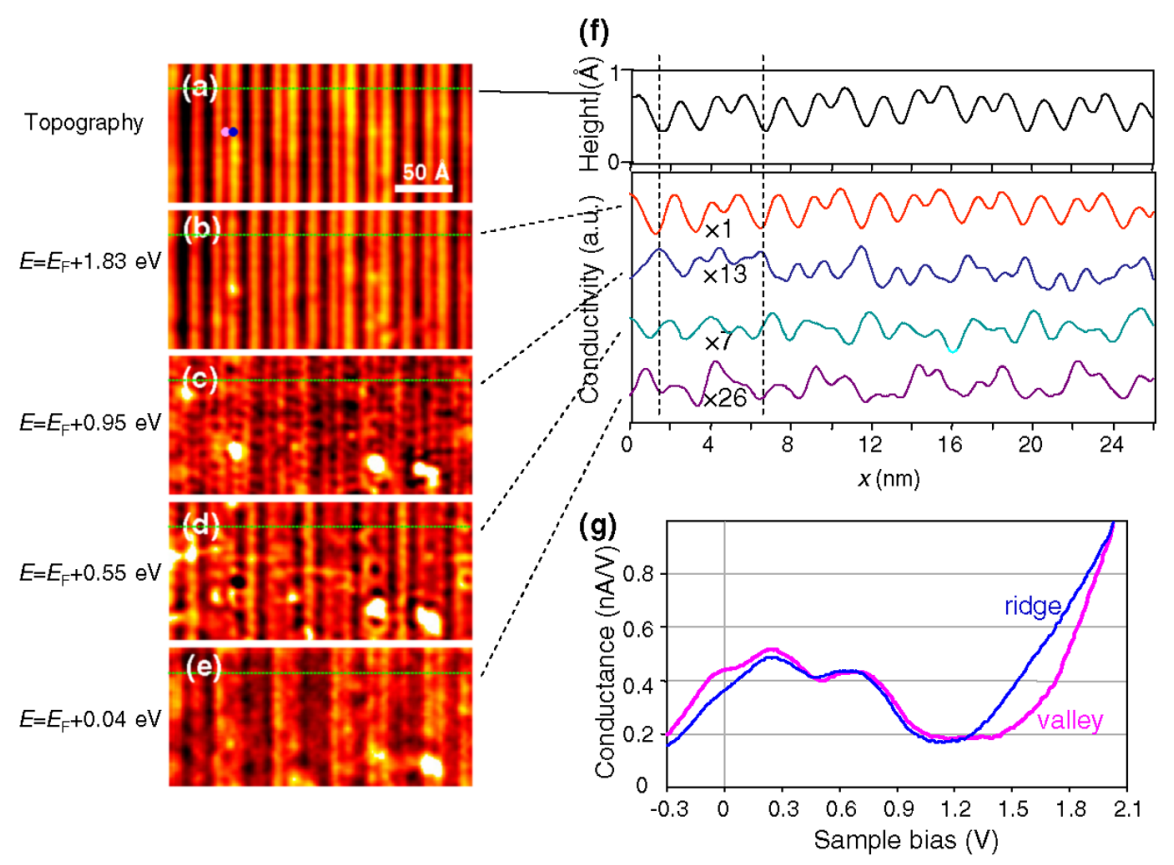

FIG. 2 (color online). (a) An STM topography of $\mathrm{Ag}$ film acquired at $6.8 \mathrm{~K}$ with sample bias $+2.3 \mathrm{~V}$. (b) -(e) The tunneling conductance images taken over the same region at the energies $1.83,0.95,0.55$, and $0.04 \mathrm{eV}$ above the Fermi level, respectively. (f) The line profiles along the horizontal lines in (a)-(e) are drawn with appropriate rescaling shown in the figure. (g) Two representative $d I / d V$ vs $V$ spectra. 
reversal (peak and valley positions are reversed). The variation of the conductance patterns as a function of energy contains the information of the $E$ vs $\boldsymbol{k}$ dispersion, which, however, is difficult to grasp directly from the real space images. In order to obtain the $\boldsymbol{k}$-resolved electronic structure, the energy-resolved conductance maps are analyzed in Fourier space.

We first discuss the energy dispersion along the periodic direction $\hat{y}$. Because of a strong energy-dependent profile along $\hat{x}$, we spatially averaged the conductance signal along $\hat{x}$. The averaging yields consistent results independent of the length of the spatial averaging. Figure 3(a) shows differential conductance spectra vs $\hat{y}$ after spatial averaging over one $L S$ unit. To enhance the contrast, a background subtraction is performed in Fig. 3(b). The result, referred to as $\rho(E, y)$, shows that with increasing energy the modulation frequency increases, leading to shorter wavelengths along $\hat{y}$. For each energy level, the Fourier transformation of $\rho(E, y)$ is calculated as $\phi\left(E, q_{y}\right)=\int \rho(E, y) e^{i q_{y} y} d y$. As discussed earlier [13,14], the spatial modulation of the LDOS with a wave vector of $q$ is a consequence of the scattering between two degenerate states $\left|E_{1}, k_{1}\right\rangle$ and $\left|E_{2}, k_{2}\right\rangle$ with $q=k_{1}-k_{2}$ and $E_{1}=E_{2}$. For a $1 \mathrm{D}$ case, this corresponds to the scattering between $|k\rangle$ and $|-k\rangle$, where $k=q / 2$. We thus define a pseudomomentum $k_{y}=q_{y} / 2$. The resulting power spectrum $\left|\phi\left(E, k_{y}\right)\right|^{2}$ is plotted in logarithmic scale in Fig. 3(c), which resembles an $E$ vs $k$ electronic structure in the extended-zone scheme. In addition, we fitted a parabolic free-electron band to the data (shown as the dashed curve along the $-k_{y}$ direction) with the band minimum at $E_{F}$ and an effective mass of $0.5 m_{0}$. This value is close to the

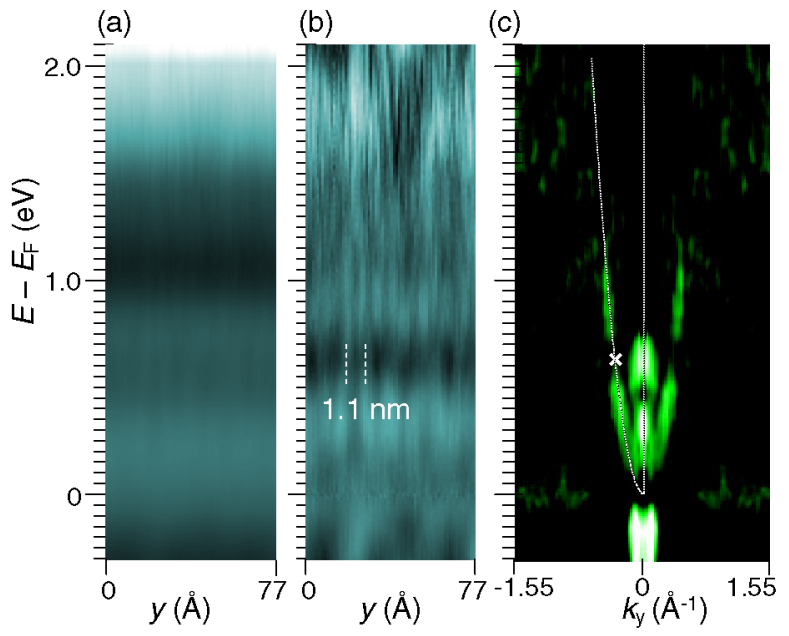

FIG. 3 (color online). (a) A spatially averaged $\operatorname{LDOS} \rho(E, y)$ obtained after averaging over an $L S$ unit along the $x$ direction. (b) The background subtracted LDOS $\rho(E, y)-\langle\rho(E)\rangle$. (c) The power spectrum $\left|\phi\left(k_{y}, E\right)\right|^{2}$ calculated from $\rho(E, y)$ and plotted in logarithmic scale. The total gray scale is from $10^{0}$ (darkest) to $10^{1.02}$ (brightest). The dashed parabola is a dispersion curve of the free electron with effective mass $0.5 m_{0}$. reported effective mass of the surface electrons on the $\operatorname{Ag}(111)$ surface $[19,20]$. Furthermore, there is an apparent gap on this free-electron-like band, at $k_{y}= \pm 0.29 \AA^{-1}$ and at $E \sim 0.65 \mathrm{eV}$ (marked by the white cross). This gap is a consequence of the periodic scattering potential with a periodicity of $11 \AA$ [see Fig. 1(b)], corresponding to the textbook case of the Bloch description of a 1D electronic structure where the gap occurs at the zone boundary at $k_{y}= \pm \pi / a$, with $a=11 \AA$.

Figure 4 illustrates the electronic structure along the quasiperiodic direction $\hat{x}$. Figure 4(a) displays an example of $\rho(E, x)$ composed of differential conductance spectra vs $x$ after spatial averaging along $\hat{y}$ over one unit cell of $11 \AA$. A background subtracted $\rho(E, x)$ with higher spatial resolution [Fig. 4(b)] reveals a quasiperiodic modulation along $\hat{x}$ at all energies. In addition, many minigaps give rise to the interferencelike fine patterns at local scale. The corresponding power spectrum of Fourier-transformed $\left|\phi\left(k_{x} E\right)\right|^{2}$ is displayed in logarithmic scale in Fig. 4(c). The Fourier space shows prominent peaks at $k_{x}$ values equal to $\frac{1}{2} k_{S}=\pi / S=0.25 \AA^{-1}, \frac{1}{2} k_{L}=\pi / L=0.18 \AA^{-1}$, and $\frac{1}{2} k_{L S}=\pi / L S=0.10 \AA^{-1}$, etc., arising from the quasiperiodic structure in real space whose basic units are $S$ and $L$. These prominent topography-related peaks mask the detailed electronic fine structure in $\left|\phi\left(k_{x} E\right)\right|^{2}$. In order to highlight the fine structure, we calculated the average power $\left\langle\left|\phi\left(k_{x}\right)\right|^{2}\right\rangle$ between the Fermi level and the topography imaging condition $\left(+2.1 \mathrm{~V}\right.$ in this case) for each $k_{x}$ and subtracted it from the original power spectrum $\left|\phi\left(k_{x}, E\right)\right|^{2}$. Indeed, the renormalized image in Fig. 4(d) removes the strong topography-related features. It further shows ubiquitous dark spots throughout this Fouriertransformed spectroscopic image, exemplifying the existence of many minigaps. Interestingly, it appears that the structures are defined by envelopes of paraboliclike bands. The existence of free-electron-like residues suggests that a macroscopically averaged technique such as angleresolved photoemission will smoothen out the fine structures in $k$ space, thus exhibiting effective but "broadened" free-electron-like structures, such as those observed for the $\mathrm{Ag} / \mathrm{GaAs}$ system [21] and the Al-Ni-Co quasicrystal [3].

Despite its complicated band structure with many minigaps, there appears to be a simple and straightforward interpretation. Take a free-electron band $e^{i k x}$ and its scattered band $e^{i(k-q) x}$ due to a Fourier component $q$ of the quasiperiodic potential. A gap pens up at $q / 2$ when $e^{i k x}$ and $e^{i(k-q) x}$ bands overlap. For example, in Fig. 4(e), the red bands are the result of scattering with the Fourier component $2 \pi / L$. Because of the quasiperiodic potential, individual Fourier components will give rise to similar effects. Figure 4(e) shows such a construction by including the effects of the first four Fourier components. One can see that it qualitatively describes the overall structure of the Fourier-transformed STS image in Fig. 4(d) rather well.

Theoretically, we have also simulated the electronic density by solving the one-dimensional Schrödinger 


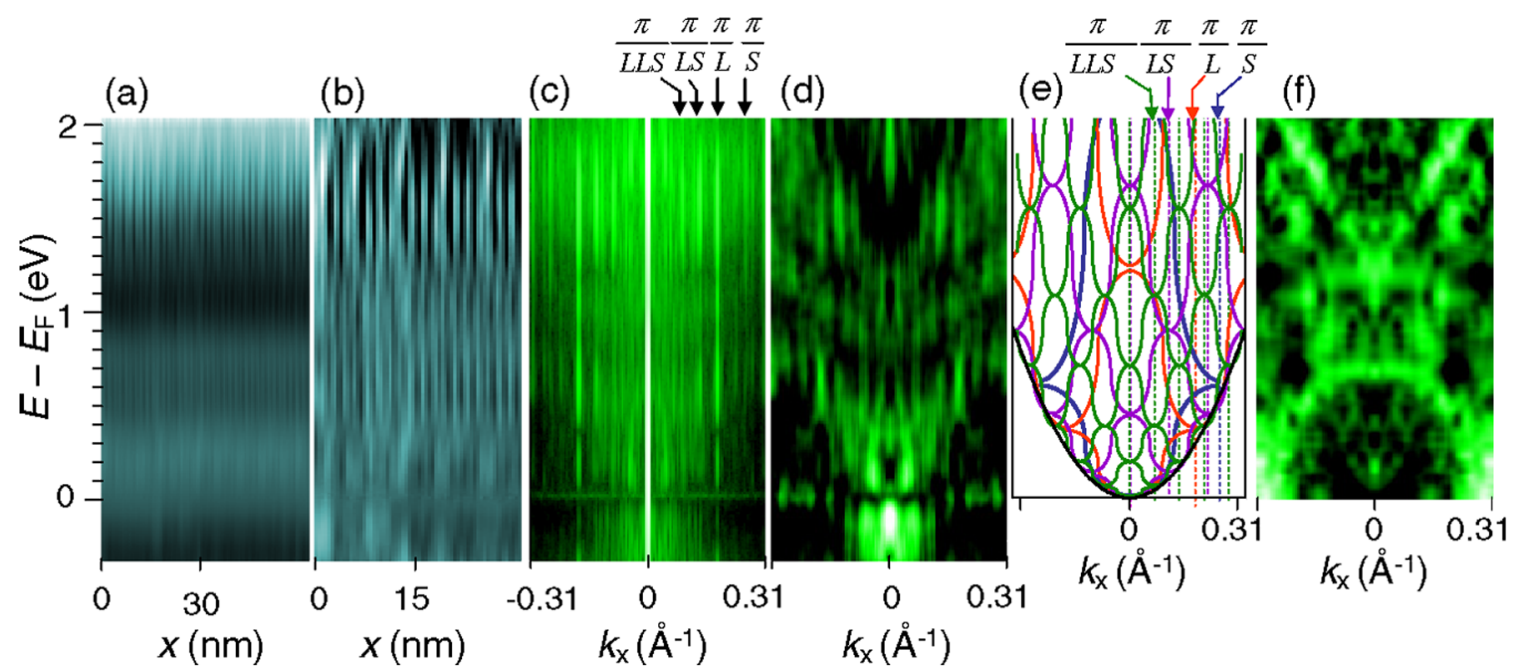

FIG. 4 (color online). (a) A spatially averaged LDOS $\rho(E, x)$ distribution. The long modulation length (11 $\AA$ ) along the $y$ direction is used as the averaging unit. (b) The zoom-in view of (a) after background subtraction. (c) The power spectrum $\left|\phi\left(k_{x}, E\right)\right|^{2}$ calculated from $\rho(E, x)$ and plotted in logarithmic scale without any background subtraction. The vertical stripe patterns occur at the characteristic feature sizes in the topography as marked by arrows. (d) The power spectrum $\left|\phi\left(k_{x}, E\right)\right|^{2}$ replotted after the background subtraction (procedure described in the text). The total gray scale is from $10^{0}$ (darkest) to $10^{1.41}$ (brightest). (e) A hypothetical band structure using an artificial construction of free-electron bands and its scattered bands (see text). (f) Typical power spectrum $\left|\phi\left(k_{x}, E\right)\right|^{2}$ calculated using a nominal scattering potential with a shape similar to the topography (see the text) and plotted with the same background subtraction procedure as that for $(\mathrm{d})$, respectively.

equation:

$$
\left[-\frac{\hbar^{2}}{2 m^{*}} \frac{\partial^{2}}{\partial x^{2}}+V(x)\right] \psi(x)=E \psi(x)
$$

where $m^{*}$ is the effective electron mass, and the potential $V(x)$ is assumed to be proportional to the topography height shown in Fig. 1(b). The energy-resolved electron density is calculated using $n_{E}(x)=\sum_{n} \delta\left(E-E_{n}\right)\left|\psi_{n}(x)\right|^{2}$, where $E_{n}$ and $\psi_{n}(x)$ are the eigenenergies and the eigenfunctions of Eq. (1), respectively. The corresponding density distribution in the $E-k$ plane is determined from the Fourier transformation of $n_{E}(x)$ over $x$ (with the experimental values $m^{*}=0.5 m_{0}$ and $E_{0}=E_{F}$ ) and, in order to illustrate the fine structures better, subjected to the same background subtraction scheme as the experimental data [Fig. 4(f)]. Although not a perfect match, the simulated result [Fig. 4(f)] captures the essential features: the existence of minigaps everywhere and free-electron-like residues. Considering that the scattering potential could not be known a priori, the qualitative agreement between the simulation and the experimental result is quite remarkable.

In summary, we have investigated the topography and spectroscopy of a silver film using a scanning tunneling microscope. The film exhibits both periodic and quasiperiodic modulations in two perpendicular directions as shown by the topographic images. Fourier analysis on the differential conductivity images reveals a Bloch electron structure in the periodic direction and an exotic spectrum with many minigaps in the quasiperiodic direction.

This work was supported by NSF DMR-0306239 and DMR-0606485.
[1] See a review by C. Janot, Quasicrystals: A Primer (Oxford University Press, New York, 1994), pp. 22-52.

[2] Ö. Rapp, in Physical Properties of Quasicrystals, edited by Z.M. Stadnik (Springer, Berlin, 1999), pp. 127-167.

[3] E. Rotenberg, W. Theis, K. Horn, and P. Gille, Nature (London) 406, 602 (2000).

[4] T. Fujiwara, in Physical Properties of Quasicrystals, edited by Z.M. Stadnik (Springer, Berlin, 1999), pp. $169-207$.

[5] Q. Niu and F. Nori, Phys. Rev. Lett. 57, 2057 (1986).

[6] F. Nori, and J.P. Rodriguez, Phys. Rev. B 34, 2207 (1986).

[7] M. Kohmoto, in Quasicrystals, edited by T. Fujiwara, and T. Ogawa (Springer, Berlin, 1990), pp. 158-168.

[8] G. E. Zil'berman, Sov. Phys. JETP 5, 208 (1957).

[9] W. Man et al., Nature (London) 436, 993 (2005).

[10] E. Abe, S. J. Pennycook, and A. P. Tsai, Nature (London) 421, 347 (2003).

[11] J. Y. Park et al., Science 309, 1354 (2005).

[12] V. Misko, S. Savel'ev, and F. Nori, Phys. Rev. Lett. 95, 177007 (2005).

[13] P. T. Sprunger et al., Science 275, 1764 (1997).

[14] K. McElroy et al., Nature (London) 422, 592 (2003).

[15] A. R. Smith et al., Science 273, 226 (1996).

[16] Ph. Ebert et al., Phys. Rev. Lett. 83, 3222 (1999).

[17] J. Tersoff and D. R. Hamann, Phys. Rev. B 31, 805 (1985).

[18] C. S. Jiang et al., Phys. Rev. B 64, 235410 (2001).

[19] S.D. Kevan and R.H. Gaylord, Phys. Rev. B 36, 5809 (1987).

[20] R. Paniago et al., Surf. Sci. 336, 113 (1995).

[21] P. Moras et al., Phys. Rev. Lett. 96, 156401 (2006). 